\title{
5th EuChemS Inorganic Chemistry Conference (EICC-5) June 24-28, 2019, Moscow
}

The 5th EuChemS Inorganic Chemistry Conference (EICC-5) will be held on June 24-28, 2019, Moscow.

Organizers

Russian Academy of Sciences (RAS)

Mendeleev Russian Chemical Society

N. S. Kurnakov Institute of General and Inorganic Chemistry of RAS

A. N. Nesmeyanov Institute of Organoelement Compounds of RAS

A. N. Frumkin Institute of Chemical Physics and Electrochemistry of RAS

Organizing committee

\section{Conference Chair Vice-Chairs}

\section{Conference Secretary}

Aslan Tsivadze

Yulia Gorbunova, Vladimir Ivanov, Alexander Trifonov Natalia Belkova

International Advisory Board

(EuChemS Inorganic Chemistry Division)

\begin{abstract}
Elisabeth Bouwman
Rik Van Deun

Milan Drabik

Roberto Gobetto

Yulia Gorbunova

Gleb Abakumov

Valentin Ananikov

Evgeny Antipov

Sergei Aldoshin

Vyacheslav Buznik

Valery Charushin

Eugeny Goodilin
\end{abstract}

$\begin{array}{ll}\text { Konrad Herbst } & \text { Laszlo Nyulaszi } \\ \text { Karl Kirchner } & \text { Luis A. Oro } \\ \text { Ana M. Martins } & \text { Gulaim Seisenbaeva } \\ \text { Dan Meyerstein } & \text { Kay Severin }\end{array}$

\section{Local Organizing Committee}

Mikhail Egorov Igor Eremenko Vladimir Fedin Stepan Kalmykov Aleksei Khokhlov Oskar Koifman Gennady Krasnikov
Vad im Kukushkin Aleksey Kuznetsov Nikolay Kuznetsov Aleksey Lukashin Valery Lunin Aziz Muzafarov
Sanja Grgurić Šipka Anna M. Trzeciak Michael Whittlesey Claudia Wickleder
Viktor Ovcharenko Valentin Sergienko Andrey Shevelkov Konstantin Solntsev Sergei Tunik Konstantin Zhizhin

\section{Conference Topics}

The conference will be organized in parallel sessions covering broadly

- Coordination and Supramolecular Chemistry

- Catalysis and Organometallic Chemistry

- Transition Metal and Main Group Chemistry

- f-Block Chemistry

- Reaction Mechanisms

- Computational Chemistry

- Medicinal and Bioinorganic Chemistry

- Magnetochemistry

- Energy and Photochemistry

- Inorganic and Organoelement Materials

The conference program will include plenary, invited and keynote lectures, oral, flash and poster presentations.

\section{Contacts:}

Conference secretary Natalia Belkova, e-mail: eicc5@ineos.ac.ru, tel.: +7 9036187745.

Coordinator of the Conference Stanislav Stegalin, e-mail: eicc5@mesol.ru, tel.: +79859601209.

\section{http://www.eicc5.ru}

